\title{
Repeated transarterial chemoembolization with epirubicin-loaded superabsorbent polymer microspheres vs. conventional transarterial chemoembolization for hepatocellular carcinoma
}

\author{
TAITO FUKUSHIMA ${ }^{1}$, MANABU MORIMOTO ${ }^{1}$, SATOSHI KOBAYASHI ${ }^{1}$, MAKOTO UENO ${ }^{1}$, YUSUKE SANO ${ }^{1}$, \\ KUNIYUKI KAWANO $^{1}$, HIROYUKI ASAMA ${ }^{1}$, SHUHEI NAGASHIMA ${ }^{1}$ and SHIN MAEDA ${ }^{2}$ \\ ${ }^{1}$ Department of Hepatobiliary and Pancreatic Oncology, Kanagawa Cancer Center, Yokohama, Kanagawa 241-8515; \\ ${ }^{2}$ Department of Gastroenterology, Yokohama City University Hospital, Yokohama, Kanagawa 236-0004, Japan
}

Received October 29, 2020; Accepted March 17, 2021

DOI: $10.3892 / \mathrm{mco} .2021 .2281$

\begin{abstract}
The aim of the present study was to evaluate the long-term outcomes and the impact of repeated conventional transarterial chemoembolization (C-TACE) and transarterial chemoembolization with epirubicin-loaded superabsorbent polymer embolics (SAP-TACE) on liver function in TACE-naïve patients with unresectable hepatocellular carcinoma (HCC). Overall, 155 consecutive patients with HCC received either C-TACE or SAP-TACE. The first cohort $(\mathrm{n}=71)$, treated between 2011 and 2014, received C-TACE; the second cohort $(\mathrm{n}=84)$, treated between 2014 and 2016, received SAP-TACE. Overall survival and deterioration of liver function were compared between the two cohorts. The 1-, 2- and 3 -year overall survival rates and median survival times were $74,50,35 \%$ and 26 months in the C-TACE cohort and 75, 60, $39 \%$ and 28 months in the SAP-TACE cohort, respectively. There were no significant differences between the two groups $(\mathrm{P}=0.289)$. Age $<70$ years, Child-Pugh class A, alpha-fetoprotein $<400 \mathrm{ng} / \mathrm{ml}$ and des-gamma-carboxy prothrombin $<1,000 \mathrm{mAU} / \mathrm{ml}$ were identified as favorable prognostic factors in multivariate analysis. In the subgroup of patients with a Child-Pugh score of 5, survival was 29 months for C-TACE vs. 55 months for SAP-TACE $(\mathrm{P}<0.05)$. In the C-TACE cohort, the median Child-Pugh score was 6 after 3 cycles and 7 after 5 cycles of TACE, and the score worsened significantly (before vs. 3 cycles, $\mathrm{P}<0.05$; before vs. 5 cycles, $\mathrm{P}<0.05$ ). In the SAP-TACE cohort, the median Child-Pugh score was 6 after 3 and 5 cycles of TACE, and the score did not worsen during the treatment cycles. There were no differences in overall survival
\end{abstract}

Correspondence to: Dr Manabu Morimoto, Department of Hepatobiliary and Pancreatic Oncology, Kanagawa Cancer Center, 2-3-2 Nakao, Asahi-ku, Yokohama, Kanagawa 241-8515, Japan E-mail: m-morimoto@kcch.jp

Key words: therapeutic chemoembolization, microsphere, hepatocellular carcinoma, drug-eluting microsphere, therapeutic embolization between repeated C-TACE and SAP-TACE in TACE-naïve patients with HCC. However, liver function deterioration was more evident in patients treated with C-TACE than in those treated with SAP-TACE.

\section{Introduction}

Hepatocellular carcinoma (HCC) is the fifth most common type of cancer worldwide and the third most common cause of cancer-related death (1). Since the majority of HCC cases occur in patients with chronic liver disease, especially cirrhosis, treatment modalities selection is determined not by tumor morphology alone, but also taking into consideration the liver function and health performance status (2). Therefore, for the HCC treatment in clinical settings, the Barcelona Clinic Liver Cancer (BCLC) staging system is used all over the world, instead of the TNM staging system used for other cancers (3).

Transarterial chemoembolization (TACE) is a widely used locoregional procedure that is recommended by several guidelines as a first-line treatment for patients with unresectable multifocal HCC (4-6). Recently, calibrated drug-eluting embolics have been developed as novel embolic devices that can overcome the drawbacks of conventional TACE (C-TACE) and enable permanent embolization effects. Several types of microspheres have been introduced for this purpose. The most commonly used embolics are DC Bead (BTG) and superabsorbent polymer embolics (SAP) (HepaSphere; Merit Medical Systems). Retrospective and prospective randomized control trials have performed a direct comparison between C-TACE and TACE with DC Bead (7-9). A meta-analysis including four randomized controlled trials and eight observational studies showed no significant differences between TACE with DC Bead and C-TACE in terms of objective response rates, survival rates, and adverse events (7).

SAP is different from other drug-eluting embolics: It is provided in a 'dry state' and, when exposed to aqueous-based media, it absorbs fluid and swells to a predictable size (10). Grosso et al (11) and Seki et al (12) reported a promising effect and a high safety profile for the treatment of HCC in their early experiences. Until recently, only a few studies have evaluated the efficacy of TACE with SAP (SAP-TACE) (13-16) compared 
with C-TACE; however, no randomized controlled trials have performed a direct comparison (17).

During the last decade, some chemotherapeutic agents have been approved for use in patients with advanced-stage HCC, especially in patients refractory to TACE therapy (18-20). These oral chemotherapeutic agents demonstrated clinical benefits in terms of survival, but they can only be administered in patients with good liver function, i.e. Child-Pugh class A. In a real clinical setting, preventing the deterioration of liver function after repeated TACE should be just as important as treatment efficacy, in the selection of the TACE procedure, because clinical trials have demonstrated the deterioration of liver function after repeated TACE $(21,22)$. However, no clinical data with repeated SAP-TACE have been reported so far.

We compared the early response and safety profile between the two TACE cohorts in the previous study (16). In the current study, we aimed to evaluate the long-term survival benefit and liver function deterioration after repeated SAP-TACE or C-TACE in larger cohorts.

\section{Materials and methods}

Study design. This retrospective, single-center study was conducted in a TACE-naïve consecutive cohort treated between January 2011 and August 2016. The study protocol was approved by our institutional review board and was conducted in accordance with local laws and the Declaration of Helsinki. All patients provided written informed consent for the treatment procedures.

Patients. Criteria to perform TACE were as follows: i) HCC diagnosis with histopathological confirmation and/or radiological examination based on the European Association for the Study of the Liver imaging criteria (3); ii) tumor location and extent not amenable to elective curative approach (resection and ablation); iii) no previous TACE or systemic chemotherapy; iv) absence of macrovascular invasion and of any suspicion of extrahepatic tumor spread; v) Child-Pugh classes A or B; vi) Eastern Cooperative Oncology Group (ECOG) performance status scores of 0 to 1 ; vii) adequate renal function (serum creatinine $<1.5$ times the upper limit of the normal range).

We administered C-TACE to consecutive patients between January 2011 and April 2014; after SAP was approved, we administered SAP-TACE to consecutive patients between May 2014 and August 2016, and we did not change the embolics choice, for the repeated use, in any case.

TACE procedure. Angiography was performed using the Seldinger technique via the femoral artery. The femoral artery was catheterized under local anesthesia, and a 4-F catheter (Selecon; Terumo) was inserted into the hepatic artery. Then, a 1.9-F microcatheter (Progreat $\Sigma$; Terumo) was advanced into the feeder arteries of each tumor.

In the C-TACE group, a mixture of $5 \mathrm{ml}$ of iodized oil (Lipiodol; Guerbet Japan) and $50 \mathrm{mg}$ of epirubicin hydrochloride (Farmorubicin; Pfizer Japan Inc.) were injected to a maximum administered dose of $50 \mathrm{mg}$, followed by embolizing with absorbable gelatin sponge particles (Gelpart; Nippon Kayaku).
In the SAP-TACE group, 50-100 $\mu \mathrm{m}$ HepaSphere microspheres were prepared as previously reported (12); 25-mg vials of embolics were preloaded with $25-30 \mathrm{mg}$ of epirubicin dissolved in $5 \mathrm{ml}$ of non-ionic contrast medium and were left unperturbed for $20 \mathrm{~min}$ to allow the embolics to expand and absorb the epirubicin after injecting the solution into the vacuum-sealed vials containing the embolics. The SAP injection was administered until near stasis (the contrast column cleared within 2-5 heartbeats) (23).

Statistical analysis. Liver function was evaluated at just before first TACE, after 3 cycles of TACE, and after 5 cycles of TACE by Child-Pugh class and albumin-bilirubin (ALBI) scores (24). Quantitative differences between the groups were analyzed using the Mann-Whitney U test, and categorical differences were analyzed using Fisher's exact or $\chi^{2}$ test. Survival time was calculated from the moment of the initial TACE. Survival curves were created using the Kaplan-Meier method and were compared using the log-rank test. Prognostic factors related to overall survival were identified by univariate and multivariate analyses. Multivariate analyses were performed using the Cox proportional hazards model to identify the independent prognostic factors. Bonferroni adjustment was used to correct for multiple comparisons. The level of significance was set up to $\mathrm{P}<0.05$. Statistical analyses were performed using SPSS version 25 software for Windows (IBM; SPSS, Inc.).

\section{Results}

Clinical characteristics. Baseline characteristics of the patients are shown in Table I. During 2011-2016, a total of 155 consecutive patients underwent TACE, including 71 patients treated with C-TACE and 84 patients treated with SAP-TACE. The follow-up period, patient age, gender, background liver disease, number of tumors, maximum tumor size, BCLC stage, Child-Pugh scores, and ALBI scores were not statistically different between the two groups. The proportion of patients that received any prior treatment before TACE was significantly different between the groups $(\mathrm{P}<0.05)$. There were no significant differences between the two groups in terms of laboratory data, the levels of aspartate aminotransferase, albumin, total bilirubin, prothrombin time, alpha-fetoprotein (AFP), and des-gamma-carboxy prothrombin (DCP).

Overall survival. We evaluated overall survival rates during follow-up for a median of 25 months. At the time of the analysis, 110 patients had died (59 in the C-TACE cohort, and 51 in the SAP-TACE cohort). Treatment-related death was not experienced. Median survival of the C-TACE cohort was 26 months, compared with 28 months for the SAP-TACE cohort (Fig. 1A). The overall survival rates were not significantly different between the two groups $(\mathrm{P}=0.289)$. The 1-, 2-, and 3-year overall survival rates were 74, 50 and $35 \%$ in the C-TACE cohort and 75, 60 and 39\% in the SAP-TACE cohort, respectively. Unilobar tumor-distribution, BCLC stage A, Child-Pugh class A, AFP $<400 \mathrm{ng} / \mathrm{ml}$ and $\mathrm{DCP}<1,000 \mathrm{mAU} / \mathrm{ml}$ were significant favorable prognostic factors in the univariate analysis. Age $<70$, Child-Pugh class A, AFP $<400 \mathrm{ng} / \mathrm{ml}$ and DCP $<1,000 \mathrm{mAU} / \mathrm{ml}$ were significant favorable prognostic factors in the multivariate Cox 
Table I. Baseline characteristics.

\begin{tabular}{|c|c|c|c|}
\hline Variable & C-TACE $(n=71)$ & SAP-TACE (n=84) & P-value \\
\hline Follow-up period, months (median; range) & $21(1-94)$ & $26(2-64)$ & 0.636 \\
\hline Age, years $($ mean $\pm S D)$ & $73.0 \pm 9.4$ & $73.4 \pm 8.7$ & 0.513 \\
\hline Sex, n (male/female) & $49 / 22$ & $61 / 23$ & 0.723 \\
\hline Etiology, n (hepatitis C/hepatitis B/other) & $30 / 11 / 30$ & $33 / 15 / 36$ & 0.898 \\
\hline Previous treatment, n (yes/no) & $6 / 65$ & $37 / 47$ & $<0.001$ \\
\hline Number of tumors & $4.7 \pm 4.9$ & $6.3 \pm 10.2$ & 0.906 \\
\hline Maximum tumor size, mm (mean $\pm \mathrm{SD})$ & $49.2 \pm 35.3$ & $43.7 \pm 38.6$ & 0.173 \\
\hline Tumor distribution, n (unilobar/bilobar) & $39 / 22$ & $40 / 29$ & 0.736 \\
\hline BCLC stage, n (A/B) & $20 / 51$ & $32 / 52$ & 0.233 \\
\hline Child-Pugh class, n (A/B) & $58 / 13$ & $59 / 24$ & 0.135 \\
\hline ALBI score, $\mathrm{n}(1 / 2 / 3)$ & $23 / 45 / 3$ & $22 / 58 / 3$ & 0.693 \\
\hline AST, IU/1 (mean \pm SD) & $71.0 \pm 90.9$ & $60.9 \pm 53.7$ & 0.245 \\
\hline Albumin, g/dl (mean \pm SD) & $3.6 \pm 0.5$ & $3.5 \pm 0.5$ & 0.567 \\
\hline Total Bilirubin, mg/dl (mean \pm SD) & $0.9 \pm 0.4$ & $0.9 \pm 0.4$ & 0.921 \\
\hline Prothrombin time, $\%($ mean $\pm \mathrm{SD})$ & $81.0 \pm 12.3$ & $78.4 \pm 12.9$ & 0.428 \\
\hline AFP, ng/ml (median; range) & $45.5(2.0-360100.0)$ & $22(1.8-111160.0)$ & 0.332 \\
\hline DCP, mAU/ml (median; range) & $151(8-400000)$ & $184(11-54400)$ & 0.803 \\
\hline
\end{tabular}

C-TACE, conventional transarterial chemoembolization; SAP-TACE, transarterial chemoembolization with superabsorbent polymer microspheres; BCLC, Barcelona Clinic Liver Cancer; AST, aspartate aminotransferase; AFP, $\alpha$-fetoprotein; DCP, des- $\gamma$-carboxy prothrombin; ALBI, albumin-bilirubin.

proportional hazard model (Table II). Treatment modality, i.e., C-TACE or SAP-TACE, did not affect long-term survival. In a subgroup analysis of patients with Child-Pugh score of 5, the SAP-TACE cohort showed longer survival compared to the C-TACE cohort $(\mathrm{P}=0.043)$ (Fig. 1B). Subgroup analysis did not show significant survival differences in patients with Child-Pugh scores of 6,7 and 8, and with BCLC stages A and B (Fig. 1C-F).

Deterioration of liver function in patients receiving C-TACE. The repeated TACE cycles were three (median) in the C-TACE cohort. In patients receiving C-TACE, median Child-Pugh scores were 5 before C-TACE, 6 after 3 cycles of C-TACE, and 7 after 5 cycles of C-TACE. The Child-Pugh scores after 3 and 5 cycle of C-TACE significantly worsened compared to those before C-TACE (before vs. 3-cycle, $\mathrm{P}=0.006$; before vs. 5 -cycle, $\mathrm{P}=0.012$ ) (left side of Fig. 2). The median ALBI scores were-2.40 before C-TACE, -2.25 after 3 cycles of C-TACE, and -1.94 after 5 cycles of C-TACE. The ALBI scores after 3 and 5 cycle C-TACE significantly worsened compared to those before C-TACE (before vs. 3-cycle, $\mathrm{P}=0.009$; before vs. 5-cycle, $\mathrm{P}=0.045$ ) (right side of Fig. 2).

Deterioration of liver function in patients receiving $S A P-T A C E$. The repeated TACE cycles were three (median) in the SAP-TACE cohort. In patients receiving SAP-TACE, Child-Pugh scores were 6 before SAP-TACE, after 3 cycles of SAP-TACE, and after 5 cycles of SAP-TACE. The Child-Pugh scores after 3 and 5 cycles of SAP-TACE did not change compared to those before SAP-TACE (left side of Fig. 3). The median ALBI scores were-2.31 before SAP-TACE, -2.45 after
3 cycles of SAP-TACE, and -2.64 after 5 cycles of SAP-TACE. The ALBI scores after 3 and 5 cycle of SAP-TACE were not statistically different compared to those before SAP-TACE (right side of Fig. 3).

Subsequent treatments. Subsequent treatments are showed in Table III. After cessation of C-TACE, 41 patients (57.7\%) received secondary treatment with radiofrequency ablation (RFA) $(n=14 ; 19.7 \%)$, surgical resection $(n=1 ; 1.4 \%)$, radiotherapy $(\mathrm{n}=2 ; 2.8 \%)$, hepatic arterial infusion chemotherapy $(\mathrm{n}=15 ; 21.1 \%)$, systemic chemotherapies $(\mathrm{n}=14 ; 19.7 \%)$. Thirty patients $(42.3 \%)$ received no secondary treatment. After cessation of SAP-TACE, 49 patients $(58.3 \%)$ received secondary treatment with RFA $(n=22 ; 26.2 \%)$, surgical resection $(n=2$; $2.4 \%)$, radiotherapy $(\mathrm{n}=5 ; 6.0 \%)$, hepatic arterial infusion chemotherapy $(n=5 ; 6.0 \%)$, systemic chemotherapies $(n=29$; $34.5 \%)$. Thirty-five patients $(41.7 \%)$ received no secondary treatment. The proportions of patients who received hepatic arterial infusion chemotherapy and systemic chemotherapies after repeated TACE were different between the C-TACE cohort and the SAP-TACE cohort $(\mathrm{P}=0.005$ and 0.004 , respectively), although those who received RFA, surgical resection, and radiotherapy were not different between the C-TACE cohort and the SAP-cohort $(\mathrm{P}=0.341,0.661$ and 0.349 , respectively).

\section{Discussion}

The current study is the first to report a comparison between the repeated use of C-TACE and SAP-TACE with respect to long-term overall survival and liver function deterioration. 
Table II. Factors associated with overall survival in all patients.

\begin{tabular}{lccc}
\hline & Univariate & Multivariate \\
\cline { 2 - 3 } Factor & P-value & P-value & Hazard ratio (95\% CI) \\
\hline Age (<70 vs. $\geq 70$ years) & 0.696 & 0.037 & $0.625(0.402-0.972)$ \\
Sex (male vs. female) & 0.566 & 0.485 & $0.831(0.494-1.397)$ \\
HCV RNA (positive vs. negative) & 0.299 & 0.434 & $0.842(0.546-1.297)$ \\
Tumor distribution (unilobar vs. bilobar) & $<0.001$ & 0.161 & $0.701(0.426-1.153)$ \\
BCLC stage (A vs. B) & $<0.001$ & 0.075 & $0.556(0.291-1.061)$ \\
Child-Pugh grade (A vs. B) & 0.002 & 0.001 & $0.427(0.256-0.714)$ \\
AFP (<400 vs. $\geq 400$ ng/ml) & $<0.001$ & 0.003 & $0.468(0.284-0.772)$ \\
DCP $(<1,000$ vs. $\geq 1,000$ mAU/ml) & $<0.001$ & 0.007 & $0.512(0.314-0.835)$ \\
TACE procedure (C-TACE vs. SAP-TACE) & 0.289 & 0.319 & $0.804(0.524-1.234)$ \\
\hline
\end{tabular}

CI, confidence interval; BCLC, Barcelona Clinic Liver Cancer; AFP, $\alpha$-fetoprotein; DCP, des- $\gamma$-carboxy prothrombin; C-TACE, conventional transarterial chemoembolization; SAP-TACE, transarterial chemoembolization with superabsorbent polymer microspheres; $\mathrm{HCV}$, hepatitis $\mathrm{C}$ virus.
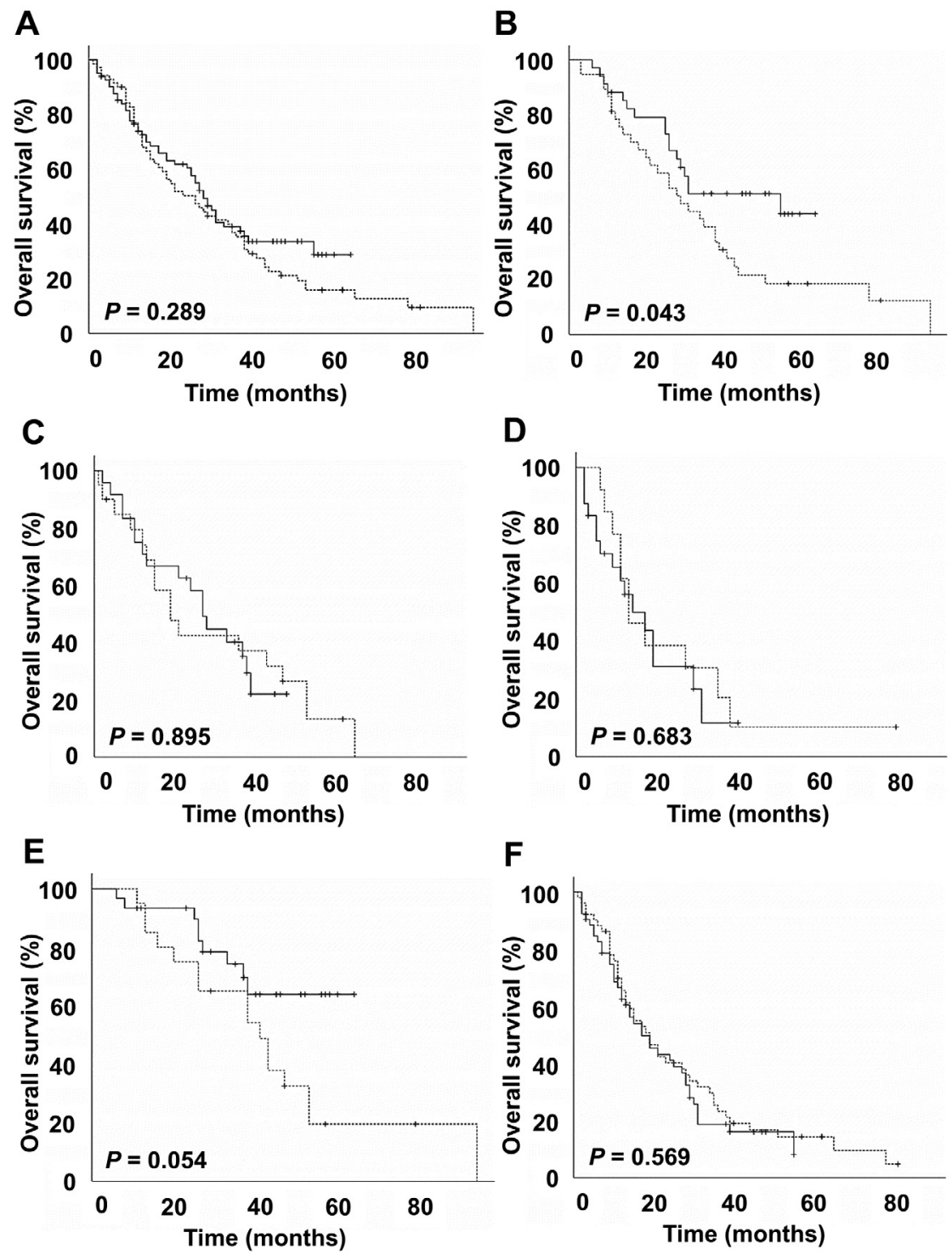

Figure 1. Survival analysis using Kaplan-Meier curves of the SAP-TACE group (continuous line) and C-TACE group (dotted line) in the various patient subgroups, including (A) all patients, (B) patients with a Child-Pugh score of 5, (C) patients with a Child-Pugh score of 6, (D) patients with a Child-Pugh score 27, (E) patients with BCLC-stage A and (F) patients with BCLC-stage B. C-TACE, conventional transarterial chemoembolization; SAP-TACE, transarterial chemoembolization with superabsorbent polymer microspheres; BCLC, Barcelona Clinic Liver Cancer. 
Table III. Subsequent treatment after TACE.

\begin{tabular}{lccr}
\hline Subsequent treatment & C-TACE $(\mathrm{n}=71)$ & SAP-TACE $(\mathrm{n}=84)$ & P-value \\
\hline RFA, n (\%) & $14(19.7)$ & $22(26.2)$ & 0.342 \\
Surgery, n (\%) & $1(1.4)$ & $2(2.4)$ & 0.661 \\
Radiation, n (\%) & $2(2.8)$ & $5(6.0)$ & 0.349 \\
Hepatic arterial infusion, n (\%) & $15(21.1)$ & $5(6.0)$ & 0.005 \\
Systemic chemotherapy, n (\%) & $14(19.7)$ & $29(34.5)$ & 0.040 \\
None, n (\%) & $30(42.3)$ & $35(41.7)$ & 0.941
\end{tabular}

C-TACE, conventional transarterial chemoembolization; SAP-TACE, transarterial chemoembolization with superabsorbent polymer microspheres; RFA, radiofrequency ablation.

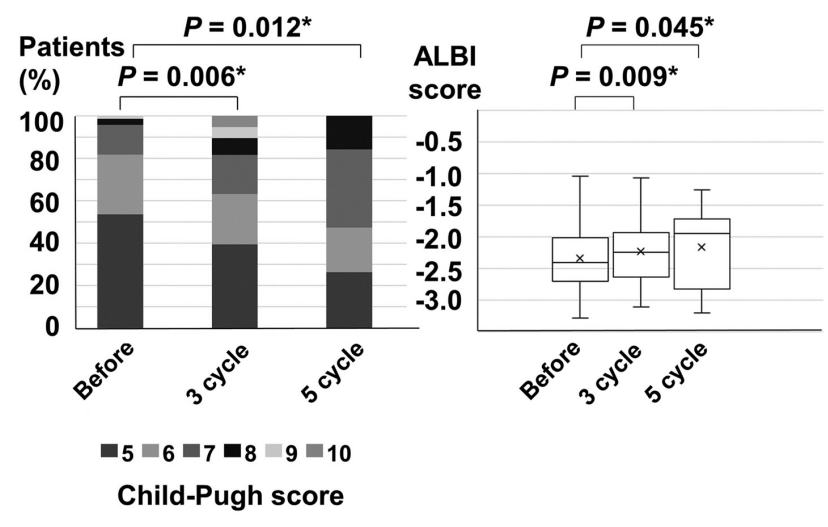

Figure 2. Liver function deterioration assessment in patients receiving repeated conventional transarterial chemoembolization. Changes in Child-Pugh scores are shown on the left. Changes in ALBI scores are shown on the right. The cross indicates the average score. ${ }^{*} \mathrm{P}<0.05$; Bonferroni adjustment was used to correct for multiple comparisons. ALBI, albumin-bilirubin.

The current study did not reveal any benefit of SAP-TACE over C-TACE in long-term survival. The median survival periods of the C-TACE cohort and the SAP-TACE cohort were 26 and 28 months respectively $(\mathrm{P}=0.289)$. However, regarding the deterioration of liver function after repeated procedures of TACE, unlike C-TACE, SAP-TACE did not worsen the Child-Pugh and ALBI scores after 3 and 5 cycles of TACE compared with the scores before TACE.

The explanation for the difference in the deterioration of liver function after the repeated administration of TACE between SAP-TACE and C-TACE may be related to the incidence of post-embolization syndrome. Preliminary results from an Italian multicenter study showed that SAP-TACE was feasible and well tolerated, with a low complication rate (11). SAP-TACE leads to low plasma levels of the cytotoxic drug and therefore minimizes toxicity compared to C-TACE (13). In a randomized controlled trial, moderate and severe periprocedural pain was less frequent after TACE with doxorubicin-eluting beads than after C-TACE (9). In a histological study, HepaSphere particles penetrated intratumoral vessels depending on their size but did not reach the hepatic sinusoids or the peribiliary plexus (10). In contrast, lipiodol spreads more distally than HepaSphere into the sinusoids and to the distal portal vein branches, allowing for a transient dual (arterial and portal) embolization (25). These results could explain why

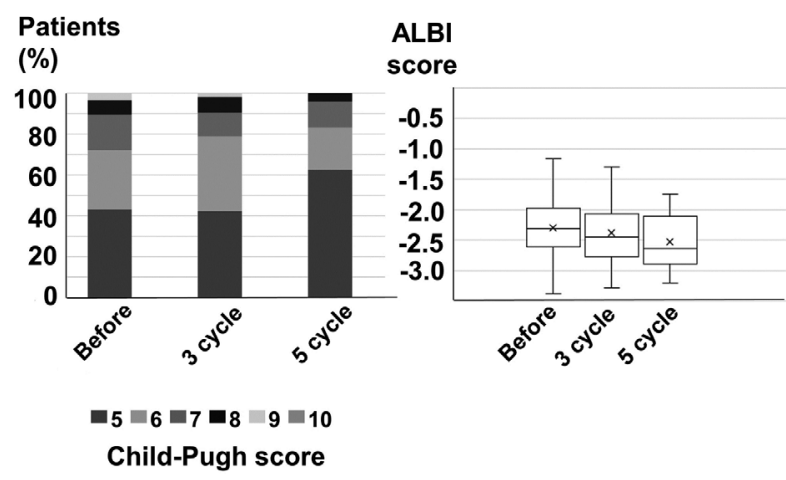

Figure 3. Liver function deterioration assessment in patients receiving repeated transarterial chemoembolization with superabsorbent polymer microspheres. Changes in Child-Pugh scores are shown on the left. Changes in ALBI scores are shown on the right. The cross indicates the average score. ALBI, albumin-bilirubin.

SAP-TACE using HepaSphere is less invasive than C-TACE using lipiodol in terms of liver function deterioration after the procedures. Another study also showed that among 99 evaluated patients, 90 (90.9\%) were not found to have direct damage to the hepatic arteries after initial SAP-TACE (12).

Recent advances allow chemotherapy with some agents in cases refractory to TACE in patients with good liver function, i.e., Child-Pugh class A (18-20). Johnson et al (24) introduced a new assessment of liver function, the ALBI grade, that can evaluate cases with excellent liver function. In patients with an excellent liver function undergoing chemotherapy, overall survival outcomes are better than in those with good or poor liver functions $(21,26,27)$. In order to 'pass the baton' to chemotherapy in TACE-refractory patients, TACE should be performed repeatedly, with no deterioration of the liver function caused by the procedures.

Although there were differences between SAP-TACE and C-TACE with respect to the deterioration of liver function after repeated TACE, they did not affect long-term outcomes. We hypothesize that the reason might lie in the treatment selection differences after refractory TACE. In this study, the choice of subsequent treatments, including hepatic arterial infusion and systemic chemotherapy, differed significantly between the two groups ( $\mathrm{P}=0.005$ and 0.04 , respectively).

There are some limitations in our study. First, the study was not randomized and includes the retrospective experience of a 
single center. Second, patients in the two cohorts had different therapeutic backgrounds, i.e., a significantly greater number of SAP-TACE patients had prior treatment as compared with C-TACE patients. Differences in biology between primary and recurrent disease can affect the efficacy of TACE. However, despite these limitations, the current study is the first to report an assessment of the choice of TACE. Taking into consideration the development of new molecular target agents and immune checkpoint inhibitors (28-30), we conclude that SAP-TACE might be an appropriate choice for preserving liver function.

\section{Acknowledgements}

Not applicable.

\section{Funding}

No funding was received.

\section{Availability of data and materials}

The datasets used and/or analyzed during the current study are available from the corresponding author on reasonable request.

\section{Authors' contributions}

TF, MM, SK, MU and SM made substantial contributions to conception and design. TF, YS, KK, HA and SN made substantial contributions to acquisition of data and/or analysis of data. TF and MM were involved in drafting the manuscript or revising it critically for important intellectual content. Each author agreed to be accountable for all aspects of the work in ensuring that questions related to the accuracy or integrity of any part of the work are appropriately investigated and resolved. All authors read and approved the final manuscript.

\section{Ethics approval and consent to participate}

The study protocol was approved by the Institutional Review Board of Kanagawa Cancer Center and was conducted in accordance with local laws and the Declaration of Helsinki. All patients provided written informed consent for the treatment procedures.

\section{Patient consent for publication}

Not applicable.

\section{Competing interests}

The authors declare that they have no competing interests.

\section{References}

1. Forner A, Reig M and Bruix J: Hepatocellular carcinoma Lancet 391: 1301-1314, 2018.

2. Vauthey JN,DixonE,AbdallaEK,Helton WS,PawlikTM,TaouliB, Brouquet A and Adams RB; American Hepato-Pancreato-Biliary Association; Society of Surgical Oncology; Society for Surgery of the Alimentary Tract: Pretreatment assessment of hepatocellular carcinoma: Expert consensus statement. HPB (Oxford) 12: 289-299, 2010
3. European Association for the Study of the Liver. Electronic address: easloffice@easloffice.eu; European Association for the Study of the Liver: EASL clinical practice guidelines: Management of hepatocellular carcinoma. J Hepatol 69: 182-236, 2018.

4. Heimbach JK, Kulik LM, Finn RS, Sirlin CB, Abecassis MM, Roberts LR, Zhu AX, Murad MH and Marrero JA: AASLD guidelines for the treatment of hepatocellular carcinoma. Hepatology 67: 358-380, 2018.

5. Omata M, Cheng AL, Kokudo N, Kudo M, Lee JM, Jia J, Tateishi R, Han KH, Chawla YK, Shiina S, et al: Asia-Pacific clinical practice guidelines on the management of hepatocellular carcinoma: A 2017 update. Hepatol Int 11: 317-370, 2017.

6. Facciorusso A, Serviddio G and Muscatiello N: Transarterial radioembolization vs. chemoembolization for hepatocarcinoma patients: A systematic review and meta-analysis. World J Hepatol 8: 770-778, 2016.

7. Facciorusso A, Di Maso M and Muscatiello N: Drug-eluting beads versus conventional chemoembolization for the treatment of unresectable hepatocellular carcinoma: A meta-analysis. Dig Liver Dis 48: 571-577, 2016.

8. Facciorusso A, Mariani L, Sposito C, Spreafico C, Bongini M, Morosi C, Cascella T, Marchianò A, Camerini T, Bhoori S, et al: Drug-eluting beads versus conventional chemoembolization for the treatment of unresectable hepatocellular carcinoma. J Gastroenterol Hepatol 31: 645-653, 2016.

9. Golfieri R, Giampalma E, Renzulli M, Cioni R, Bargellini I, Bartolozzi C, Breatta AD, Gandini G, Nani R, Gasparini D, et al: Randomised controlled trial of doxorubicin-eluting beads vs. conventional chemoembolisation for hepatocellular carcinoma. Br J Cancer 111: 255-264, 2014.

10. Osuga K, Khankan AA, Hori S, Okada A, Sugiura T, Maeda M, Nagano $H$, Yamada A, Murakami $T$ and Nakamura $H$ : Transarterial embolization for large hepatocellular carcinoma with use of superabsorbent polymer microspheres: Initial experience. J Vasc Interv Radiol 13: 929-934, 2002.

11. Grosso M, Vignali C, Quaretti P, Nicolini A, Melchiorre F, Gallarato G, Bargellini I, Petruzzi P, Massa Saluzzo C, Crespi S and Sarti I: Transarterial chemoembolization for hepatocellular carcinoma with drug-eluting microspheres: Preliminary results from an Italian multicentre study. Cardiovasc Intervent Radiol 31: 1141-1149, 2008.

12. Seki A, Hori S, Kobayashi K and Narumiya S: Transcatheter arterial chemoembolization with epirubicin-loaded superabsorbent polymer microspheres for 135 hepatocellular carcinoma patients: Single-center experience. Cardiovasc Intervent Radiol 34: 557-565, 2011.

13. van Malenstein H, Maleux G, Vandecaveye V, Heye S, Laleman W, van Pelt J, Vaninbroukx J, Nevens F and Verslype C: A randomized phase II study of drug-eluting beads versus transarterial chemoembolization for unresectable hepatocellular carcinoma. Onkologie 34: 368-376, 2011.

14. Duan F, Wang EQ, Lam MG, Abdelmaksoud MH, Louie JD, Hwang GL, Kothary N, Kuo WT, Hofmann LV and Sze DY: Superselective chemoembolization of HCC: Comparison of short-term safety and efficacy between drug-eluting LC beads, quadraspheres, and conventional ethiodized oil emulsion. Radiology 278: 612-621, 2016.

15. Kucukay F, Badem S, Karan A, Ozdemir M, Okten RS, Ozbulbul NI, Kucukay MB, Unlu I, Bostanci EB and Akdogan M: A single-center retrospective comparison of doxorubicin-loaded hepasphere transarterial chemoembolization with conventional transarterial chemoembolization for patients with unresectable hepatocellular carcinoma. J Vasc Interv Radiol 26: 1622-1629, 2015.

16. Morimoto M, Kobayashi S, Moriya S, Ueno M, Tezuka S, Irie K, Goda Y and Ohkawa S: Short-term efficacy of transarterial chemoembolization with epirubicin-loaded superabsorbent polymer microspheres for hepatocellular carcinoma: Comparison with conventional transarterial chemoembolization. Abdom Radiol (NY) 42: 612-619, 2017.

17. Facciorusso A: Drug-eluting beads transarterial chemoembolization for hepatocellular carcinoma: Current state of the art. World J Gastroenterol 24: 161-169, 2018.

18. Bruix J, Qin S, Merle P, Granito A, Huang YH, Bodoky G, Pracht M, Yokosuka O, Rosmorduc O, Breder V, et al: Regorafenib for patients with hepatocellular carcinoma who progressed on sorafenib treatment (RESORCE): A randomised, double-blind, placebo-controlled, phase 3 trial. Lancet 389: 56-66, 2017. 
19. Kudo M, Finn RS, Qin S, Han KH, Ikeda K, Piscaglia F, Baron A, Park JW, Han G, Jassem J, et al: Lenvatinib versus sorafenib in first-line treatment of patients with unresectable hepatocellular carcinoma: A randomised phase 3 non-inferiority trial. Lancet 391: 1163-1173, 2018.

20. Llovet JM, Ricci S, Mazzaferro V, Hilgard P, Gane E, Blanc JF, de Oliveira AC, Santoro A, Raoul JL, Forner A, et al: Sorafenib in advanced hepatocellular carcinoma. N Engl J Med 359: 378-390, 2008

21. Hiraoka A, Kumada T, Kudo M, Hirooka M, Koizumi Y, Hiasa Y, Tajiri K, Toyoda H, Tada T, Ochi H, et al: Hepatic function during repeated TACE procedures and prognosis after introducing sorafenib in patients with unresectable hepatocellular carcinoma: Multicenter analysis. Dig Dis 35: 602-610, 2017.

22. Miksad RA, Ogasawara S, Xia F, Fellous M and Piscaglia F: Liver function changes after transarterial chemoembolization in US hepatocellular carcinoma patients: The LiverT study. BMC Cancer 19: 795, 2019.

23. Lencioni R, de Baere T, Burrel M, Caridi JG, Lammer J, Malagari K, Martin RC, O'Grady E, Real MI, Vogl TJ, et al: Transcatheter treatment of hepatocellular carcinoma with doxorubicin-loaded DC Bead (DEBDOX): Technical recommendations. Cardiovasc Intervent Radiol 35: 980-985, 2012.

24. Johnson PJ, Berhane S, Kagebayashi C, Satomura S, Teng M, Reeves HL, O'Beirne J, Fox R, Skowronska A, Palmer D, et al: Assessment of liver function in patients with hepatocellular carcinoma: A new evidence-based approach-the ALBI grade. J Clin Oncol 33: 550-558, 2015.

25. Idee JM and Guiu B: Use of Lipiodol as a drug-delivery system for transcatheter arterial chemoembolization of hepatocellular carcinoma: A review. Crit Rev Oncol Hematol 88: 530-549, 2013.
26. Pinato DJ, Yen C, Bettinger D, Ramaswami R, Arizumi T, Ward C, Pirisi M, Burlone ME, Thimme R, Kudo M and Sharma R: The albumin-bilirubin grade improves hepatic reserve estimation post-sorafenib failure: Implications for drug development. Aliment Pharmacol Ther 45: 714-722, 2017.

27. Ueshima K, Nishida N, Hagiwara S, Aoki T, Minami T, Chishina $\mathrm{H}$, Takita M, Minami Y, Ida $\mathrm{H}$, Takenaka M, et al: Impact of baseline ALBI grade on the outcomes of hepatocellular carcinoma patients treated with lenvatinib: A multicenter study. Cancers (Basel) 11: 952, 2019.

28. El-Khoueiry AB, Sangro B, Yau T, Crocenzi TS, Kudo M, Hsu C, Kim TY, Choo SP, Trojan J, Welling TH Rd, et al: Nivolumab in patients with advanced hepatocellular carcinoma (CheckMate 040): An open-label, non-comparative, phase $1 / 2$ dose escalation and expansion trial. Lancet 389: 2492-2502, 2017.

29. Abou-Alfa GK, Meyer T, Cheng AL, El-Khoueiry AB, Rimassa L, Ryoo BY, Cicin I, Merle P, Chen Y, Park JW, et al: Cabozantinib in patients with advanced and progressing hepatocellular carcinoma. N Engl J Med 379: 54-63, 2018.

30. Zhu AX, Finn RS, Edeline J, Cattan S, Ogasawara S, Palmer D, Verslype C,Zagonel V, Fartoux L, Vogel A, et al: Pembrolizumab in patients with advanced hepatocellular carcinoma previously treated with sorafenib (KEYNOTE-224): A non-randomised, open-label phase 2 trial. Lancet Oncol 19: 940-952, 2018.

This work is licensed under a Creative Commons

Attribution-NonCommercial-NoDerivatives 4.0 International (CC BY-NC-ND 4.0) License. 